\title{
GROUP CLASSIFICATION FOR A GENERAL NONLINEAR MODEL OF OPTION PRICING ${ }^{1}$
}

\author{
Vladimir E. Fedorov \\ Laboratory of Quantum Topology, Mathematical Analysis Department, Chelyabinsk State \\ University, Chelyabinsk, Russia, kar@csu.ru
}

Mikhail M. Dyshaev

Mathematical Analysis Department, Chelyabinsk State University, Chelyabinsk, Russia, MikhailDyshaev@gmail.com

\begin{abstract}
We consider a family of equations with two free functional parameters containing the classical Black-Scholes model, Schönbucher-Wilmott model, Sircar-Papanicolaou equation for option pricing as partial cases. A five-dimensional group of equivalence transformations is calculated for that family. That group is applied to a search for specifications' parameters specifications corresponding to additional symmetries of the equation. Seven pairs of specifications are found.

Key words: Nonlinear partial differential equation, Group analysis, Group of equivalency transformations, Group classification, Nonlinear Black-Scholes equation, Pricing options, Dynamic hedging, Feedback effects of hedging.
\end{abstract}

\section{Introduction}

In the paper a nonlinear model

$$
u_{t}+\frac{w(t, x) u_{x x}}{2\left(1-x v\left(u_{x}\right) u_{x x}\right)^{2}}+r\left(x u_{x}-u\right)=0
$$

from the theory of financial markets is considered. In the case of $v \equiv 0$ it is generalized BlackScholes equation [1], if, besides, $w(t, x)=\sigma^{2} x^{2}(0.1)$ is the classical Black-Scholes model [2]. For arbitrary $v$ and $w(t, x)=\sigma^{2} x^{2}(0.1)$ is the Sircar-Papanicolaou nonlinear feedback pricing equation [1]. If $v$ is arbitrary, $w(t, x)=\sigma^{2} x^{2}$ and $r=0$, it is the equilibrium pricing model or Schönbucher-Wilmott nonlinear feedback pricing model [3-6]. The last two models take into account a feedback effect of the presence of two types of traders. The programm traders are the portfolio insurers and the reference traders are the Black - Scholes uploaders.

The aim of the paper is to obtain a group classification [7] of equation (0.1) with free parameters $v$ and $w$. The group of equivalence transformations $[7,8]$ of equation $(0.1)$ will be found. By means of this group symmetries for the equation with all specifications will be calculated. Further these results will be applied to the theory of financial markets, particularly, they will allow to calculate various exact solutions of equation (0.1).

The groups of classical Black - Scholes model and their accordance to the groups of the heat equation were found in [9]. Research of symmetries of Schönbucher-Wilmott model and of some other nonlinear pricing models was made in [10-13].

\footnotetext{
${ }^{1}$ The work is partially supported by Laboratory of Quantum Topology of Chelyabinsk State University (Russian Federation government grant 14.Z50.31.0020).
} 


\section{Group of the equivalence transformations}

Let us find the continuous group of equivalence transformations of equation (0.1) for the applying to the search of specifications of the functions $v=v\left(u_{x}\right), w=w(t, x)$ in the equation, that corresponds to additional symmetries for the symmetries of the kernel of principal Lie group for the equation. We rewrite equation (0.1) in the form

$$
u_{t}+\frac{w u_{x x}}{2\left(1-x v u_{x x}\right)^{2}}+r\left(x u_{x}-u\right)=0,
$$

where $v, w$ are the additional variables, depending on $t, x, u, u_{t}$ and $u_{x}$. Generators of a continuous group of equivalence transformations will be searched in the form $Y=\tau \partial_{t}+\xi \partial_{x}+\eta \partial_{u}+\mu \partial_{v}+\nu \partial_{w}$, where the functions $\tau, \xi, \eta$ depend on $t, x, u$, and $\mu \nu$ depend on $t, x, u, u_{t}, u_{x}, v, w$. For brevity hereafter $\frac{\partial}{\partial t} \equiv \partial_{t}$ and similar notations are used. We add to (1.1) the equations

$$
\begin{gathered}
v_{t}=0, \quad v_{x}=0, \quad v_{u}=0, \quad v_{u_{t}}=0, \\
w_{u}=0, \quad w_{u_{t}}=0, \quad w_{u_{x}}=0,
\end{gathered}
$$

meaning that in the statement of the problem the function $v$ depends only on $u_{x}$ and the function $w$ depends on $t, x$.

We consider the system of equations (1.1)-(1.3) as a manifold $\mathfrak{N}$ in an expanded space of corresponding variables. Let us act on the left-hand side of system (1.1)-(1.3) by the extended operator

$$
\tilde{Y}=Y+\varphi^{t} \partial_{u_{t}}+\varphi^{x x} \partial_{u_{x x}}+\mu^{t} \partial_{v_{t}}+\mu^{x} \partial_{v_{x}}+\mu^{u} \partial_{v_{u}}+\mu^{u_{t}} \partial_{v_{u_{t}}}+\nu^{u} \partial_{w_{u}}+\nu^{u_{t}} \partial_{w_{u_{t}}}+\nu^{u_{x}} \partial_{w_{u_{x}}},
$$

we restrict a result of the action on $\mathfrak{N}$ and we obtain the equations

$$
\begin{gathered}
\varphi^{t}+\frac{v w u_{x x}^{2} \xi}{\left(1-x v u_{x x}\right)^{3}}+\frac{w\left(1+x v u_{x x}\right) \varphi^{x x}}{2\left(1-x v u_{x x}\right)^{3}}+\frac{u_{x x} \nu}{2\left(1-x v u_{x x}\right)^{2}}+\frac{w x u_{x x}^{2} \mu}{\left(1-x v u_{x x}\right)^{3}}+ \\
+\left.r\left(u_{x} \xi+x \varphi^{x}-\eta\right)\right|_{\mathfrak{N}}=0, \\
\left.\mu^{t}\right|_{\mathfrak{N}}=0,\left.\quad \mu^{x}\right|_{\mathfrak{N}}=0,\left.\quad \mu^{u}\right|_{\mathfrak{N}}=0,\left.\quad \mu^{u_{t}}\right|_{\mathfrak{N}}=0, \\
\left.\nu^{u}\right|_{\mathfrak{N}}=0,\left.\quad \nu^{u_{t}}\right|_{\mathfrak{N}}=0,\left.\quad \mu^{u_{x}}\right|_{\mathfrak{N}}=0 .
\end{gathered}
$$

From (1.2) and (1.3) it follows that

$$
\begin{gathered}
\widetilde{D}_{t}=\partial_{t}+w_{t} \partial_{w}+w_{t t} \partial_{w_{t}}+w_{t x} \partial_{w_{x}}+\ldots, \quad \widetilde{D}_{x}=\partial_{x}+w_{x} \partial_{w}+w_{t x} \partial_{w_{t}}+w_{x x} \partial_{w_{x}}+\ldots, \\
\widetilde{D}_{u}=\partial_{u}, \quad \widetilde{D}_{u_{t}}=\partial_{u_{t}}, \quad \widetilde{D}_{u_{x}}=\partial_{u_{x}}+v^{\prime}\left(u_{x}\right) \partial_{v}+v^{\prime \prime}\left(u_{x}\right) \partial_{v^{\prime}\left(u_{x}\right)}+\ldots, \\
\mu^{t}=\mu_{t}+w_{t} \mu_{w}-v^{\prime}\left(u_{x}\right) \varphi_{t}^{x}=\mu_{t}+w_{t} \mu_{w}-v^{\prime}\left(u_{x}\right)\left(\eta_{t x}+u_{x} \eta_{t u}-u_{t} \tau_{t x}-u_{t} u_{x} \tau_{t u}-u_{x} \xi_{t x}-u_{x}^{2} \xi_{t u}\right), \\
\mu^{x}=\mu_{x}+w_{x} \mu_{w}-v^{\prime}\left(u_{x}\right) \varphi_{x}^{x}=\mu_{x}+w_{x} \mu_{w}-v^{\prime}\left(u_{x}\right)\left(\eta_{x x}+u_{x} \eta_{x u}-u_{t} \tau_{x x}-u_{t} u_{x} \tau_{x u}-u_{x} \xi_{x x}-u_{x}^{2} \xi_{x u}\right), \\
\mu^{u}=\mu_{u}-v^{\prime}\left(u_{x}\right) \varphi_{u}^{x}=\mu_{u}-v^{\prime}\left(u_{x}\right)\left(\eta_{x u}+u_{x} \eta_{u u}-u_{t} \tau_{x u}-u_{t} u_{x} \tau_{u u}-u_{x} \xi_{x u}-u_{x}^{2} \xi_{u u}\right), \\
\mu^{u t}=\mu_{u_{t}}-v^{\prime}\left(u_{x}\right) \varphi_{u_{t}}^{x}=\mu_{u_{t}}+v^{\prime}\left(u_{x}\right) \tau_{x}+u_{x} v^{\prime}\left(u_{x}\right) \tau_{u}, \\
\nu^{u}=\nu_{u}-w_{t} \tau_{u}-w_{x} \xi_{u}, \quad \nu^{u t}=\nu_{u_{t}}, \quad \nu^{u_{x}}=\nu_{u_{x}}+v^{\prime}\left(u_{x}\right) \nu_{v} .
\end{gathered}
$$

Therefore, equations (1.5) and (1.6) have the form

$$
\mu_{t}+w_{t} \mu_{w}-\left.v^{\prime}\left(u_{x}\right)\left(\eta_{t x}+u_{x} \eta_{t u}-u_{t} \tau_{t x}-u_{t} u_{x} \tau_{t u}-u_{x} \xi_{t x}-u_{x}^{2} \xi_{t u}\right)\right|_{\mathfrak{N}}=0,
$$




$$
\begin{gathered}
\mu_{x}+w_{x} \mu_{w}-\left.v^{\prime}\left(u_{x}\right)\left(\eta_{x x}+u_{x} \eta_{x u}-u_{t} \tau_{x x}-u_{t} u_{x} \tau_{x u}-u_{x} \xi_{x x}-u_{x}^{2} \xi_{x u}\right)\right|_{\mathfrak{N}}=0 \\
\mu_{u}-\left.v^{\prime}\left(u_{x}\right)\left(\eta_{x u}+u_{x} \eta_{u u}-u_{t} \tau_{x u}-u_{t} u_{x} \tau_{u u}-u_{x} \xi_{x u}-u_{x}^{2} \xi_{u u}\right)\right|_{\mathfrak{N}}=0 \\
\mu_{u_{t}}+v^{\prime}\left(u_{x}\right) \tau_{x}+u_{x} v^{\prime}\left(u_{x}\right) \tau_{u}=0 \\
\nu_{u}-w_{t} \tau_{u}-w_{x} \xi_{u}=0, \quad \nu_{u_{t}}=0, \quad \nu_{u_{x}}+v^{\prime}\left(u_{x}\right) \nu_{v}=0
\end{gathered}
$$

By equality (1.1) equations (1.7)-(1.9) can be rewritten in the form

$$
\begin{gathered}
\mu_{t}+w_{t} \mu_{w}-v^{\prime}\left(u_{x}\right)\left(\eta_{t x}+u_{x} \eta_{t u}-u_{x} \xi_{t x}-u_{x}^{2} \xi_{t u}+\right. \\
\left.+\frac{w u_{x x}\left(\tau_{t x}+u_{x} \tau_{t u}\right)}{2\left(1-x v u_{x x}\right)^{2}}+\left(r x u_{x}-r u\right)\left(\tau_{t x}+u_{x} \tau_{t u}\right)\right)=0 \\
\mu_{x}+w_{x} \mu_{w}-v^{\prime}\left(u_{x}\right)\left(\eta_{x x}+u_{x} \eta_{x u}-u_{x} \xi_{x x}-u_{x}^{2} \xi_{x u}+\right. \\
\left.+\frac{w u_{x x}\left(\tau_{x x}+u_{x} \tau_{x u}\right)}{2\left(1-x v u_{x x}\right)^{2}}+\left(r x u_{x}-r u\right)\left(\tau_{x x}+u_{x} \tau_{x u}\right)\right)=0, \\
\mu_{u}-v^{\prime}\left(u_{x}\right)\left(\eta_{x u}+u_{x} \eta_{u u}-u_{x} \xi_{x u}-u_{x}^{2} \xi_{u u}+\right. \\
\left.+\frac{w u_{x x}\left(\tau_{x u}+u_{x} \tau_{u u}\right)}{2\left(1-x v u_{x x}\right)^{2}}+\left(r x u_{x}-r u\right)\left(\tau_{x u}+u_{x} \tau_{u u}\right)\right)=0 .
\end{gathered}
$$

By means of the equality

$$
\begin{aligned}
\varphi^{x x} & =\eta_{x x}+2 u_{x} \eta_{x u}+u_{x}^{2} \eta_{u u}+u_{x x} \eta_{u}-u_{t} \tau_{x x}-2 u_{t} u_{x} \tau_{x u}-2 u_{t x} \tau_{x}-u_{t} u_{x}^{2} \tau_{u u}- \\
& -2 u_{x} u_{t x} \tau_{u}-u_{t} u_{x x} \tau_{u}-u_{x} \xi_{x x}-2 u_{x}^{2} \xi_{x u}-2 u_{x x} \xi_{x}-u_{x}^{3} \xi_{u u}-3 u_{x} u_{x x} \xi_{u}
\end{aligned}
$$

equation (1.4) is rewritten as

$$
\begin{aligned}
& \eta_{t}+u_{t} \eta_{u}-u_{t} \tau_{t}-u_{t}^{2} \tau_{u}-u_{x} \xi_{t}-u_{t} u_{x} \xi_{u}+\frac{1}{2\left(1-x v u_{x x}\right)^{3}}\left(2 v w u_{x x}^{2} \xi+2 x w u_{x x}^{2} \mu+\right. \\
& +u_{x x} \nu-x u_{x x}^{2} v \nu+w\left(1+x v u_{x x}\right)\left(\eta_{x x}+2 u_{x} \eta_{x u}+u_{x}^{2} \eta_{u u}+u_{x x} \eta_{u}-\right. \\
& -u_{t} \tau_{x x}-2 u_{t} u_{x} \tau_{x u}-2 u_{t x} \tau_{x}-u_{t} u_{x}^{2} \tau_{u u}-2 u_{x} u_{t x} \tau_{u}- \\
& \left.\left.-u_{t} u_{x x} \tau_{u}-u_{x} \xi_{x x}-2 u_{x}^{2} \xi_{x u}-2 u_{x x} \xi_{x}-u_{x}^{3} \xi_{u u}-3 u_{x} u_{x x} \xi_{u}\right)\right)+ \\
& +r u_{x} \xi+r x\left(\eta_{x}+u_{x} \eta_{u}-u_{t} \tau_{x}-u_{t} u_{x} \tau_{u}-u_{x} \xi_{x}-u_{x}^{2} \xi_{u}\right)-\left.r \eta\right|_{\mathfrak{N}}= \\
& =\eta_{t}+\frac{w u_{x x}\left(\tau_{t}-\eta_{u}\right)}{2\left(1-x v u_{x x}\right)^{2}}+\left(r x u_{x}-r u\right)\left(\tau_{t}-\eta_{u}\right)-\frac{w^{2} u_{x x}^{2} \tau_{u}}{4\left(1-x v u_{x x}\right)^{4}}- \\
& -\left(r x u_{x}-r u\right)^{2} \tau_{u}+\frac{w u_{x x}\left(r x u_{x}-r u\right) \tau_{u}}{\left(1-x v u_{x x}\right)^{2}}-u_{x} \xi_{t}+ \\
& +\frac{w u_{x} u_{x x} \xi_{u}}{2\left(1-x v u_{x x}\right)^{2}}+\left(r x u_{x}-r u\right) u_{x} \xi_{u}+\frac{1}{2\left(1-x v u_{x x}\right)^{3}}\left(2 v w u_{x x}^{2} \xi+2 x w u_{x x}^{2} \mu+\right. \\
& +u_{x x} \nu-x u_{x x}^{2} v \nu+w\left(1+x v u_{x x}\right)\left(\eta_{x x}+2 u_{x} \eta_{x u}+u_{x}^{2} \eta_{u u}+u_{x x} \eta_{u}+\right. \\
& +\frac{w u_{x x} \tau_{x x}}{2\left(1-x v u_{x x}\right)^{2}}+\left(r x u_{x}-r u\right) \tau_{x x}+\frac{w u_{x} u_{x x} \tau_{x u}}{\left(1-x v u_{x x}\right)^{2}}+2\left(r x u_{x}-r u\right) u_{x} \tau_{x u}- \\
& -2 u_{t x} \tau_{x}+\frac{w u_{x}^{2} u_{x x} \tau_{u u}}{2\left(1-x v u_{x x}\right)^{2}}+\left(r x u_{x}-r u\right) u_{x}^{2} \tau_{u u}-2 u_{x} u_{t x} \tau_{u}+\frac{w u_{x x}^{2} \tau_{u}}{2\left(1-x v u_{x x}\right)^{2}}+ \\
& \left.\left.+\left(r x u_{x}-r u\right) u_{x x} \tau_{u}-u_{x} \xi_{x x}-2 u_{x}^{2} \xi_{x u}-2 u_{x x} \xi_{x}-u_{x}^{3} \xi_{u u}-3 u_{x} u_{x x} \xi_{u}\right)\right)+ \\
& +r u_{x} \xi+r x\left(\eta_{x}+u_{x} \eta_{u}-u_{x} \xi_{x}-u_{x}^{2} \xi_{u}\right)-r \eta+ \\
& +\frac{r x w u_{x x}\left(\tau_{x}+u_{x} \tau_{u}\right)}{2\left(1-x v u_{x x}\right)^{2}}+r x\left(r x u_{x}-r u\right)\left(\tau_{x}+u_{x} \tau_{u}\right)=0 .
\end{aligned}
$$


We differentiate the last equations with respect to $u_{t x}$ and obtain $w\left(1+x v u_{x x}\right)\left(\tau_{x}+u_{x} \tau_{u}\right)=0$, consequently, $\tau=\tau(t)$, if $w \neq 0$. Therefore, equations (1.11)-(1.15) have the form

$$
\begin{gathered}
\mu_{u_{t}}=0, \quad \nu_{u}-w_{x} \xi_{u}=0, \quad \nu_{u_{t}}=0, \quad \nu_{u_{x}}+v^{\prime}\left(u_{x}\right) \nu_{v}=0 \\
\mu_{t}+w_{t} \mu_{w}-v^{\prime}\left(u_{x}\right)\left(\eta_{t x}+u_{x} \eta_{t u}-u_{x} \xi_{t x}-u_{x}^{2} \xi_{t u}\right)=0 \\
\mu_{x}+w_{x} \mu_{w}-v^{\prime}\left(u_{x}\right)\left(\eta_{x x}+u_{x} \eta_{x u}-u_{x} \xi_{x x}-u_{x}^{2} \xi_{x u}\right)=0 \\
\mu_{u}-v^{\prime}\left(u_{x}\right)\left(\eta_{x u}+u_{x} \eta_{u u}-u_{x} \xi_{x u}-u_{x}^{2} \xi_{u u}\right)=0 \\
\eta_{t}+\frac{w u_{x x}\left(\tau^{\prime}(t)-\eta_{u}\right)}{2\left(1-x v u_{x x}\right)^{2}}+\left(r x u_{x}-r u\right)\left(\tau^{\prime}(t)-\eta_{u}\right)-u_{x} \xi_{t}+ \\
+\frac{1}{2\left(1-x v u_{x} u_{x x} \xi_{u}\right)^{2}}+\left(r x u_{x}-r u\right) u_{x} \xi_{u}+\frac{1}{2\left(1-x v u_{x x}\right)^{3}}\left(2 v w u_{x x}^{2} \xi+2 x w u_{x x}^{2} \mu+\right. \\
+u_{x x} \nu-x u_{x x}^{2} v \nu+w\left(1+x v u_{x x}\right)\left(\eta_{x x}+2 u_{x} \eta_{x u}+u_{x}^{2} \eta_{u u}+u_{x x} \eta_{u}-u_{x} \xi_{x x}-\right. \\
\left.\left.-2 u_{x}{ }^{2} \xi_{x u}-2 u_{x x} \xi_{x}-u_{x}^{3} \xi_{u u}-3 u_{x} u_{x x} \xi_{u}\right)\right)+r u_{x} \xi+ \\
+r x\left(\eta_{x}+u_{x} \eta_{u}-u_{x} \xi_{x}-u_{x}^{2} \xi_{u}\right)-r \eta=0
\end{gathered}
$$

We multiply by $2\left(1-x v u_{x x}\right)^{3}$ the last equation, then

$$
\begin{gathered}
2\left(1-x v u_{x x}\right)^{3}\left(\eta_{t}+\left(r x u_{x}-r u\right)\left(\tau^{\prime}(t)-\eta_{u}+u_{x} \xi_{u}\right)\right)+\left(1-x v u_{x x}\right) w u_{x x}\left(\tau^{\prime}(t)-\eta_{u}\right)- \\
-2\left(1-x v u_{x x}\right)^{3} u_{x} \xi_{t}+\left(1-x v u_{x x}\right) w u_{x} u_{x x} \xi_{u}+2 v w u_{x x}^{2} \xi+2 x w u_{x x}^{2} \mu+ \\
+u_{x x} \nu-x u_{x x}^{2} v \nu+w\left(1+x v u_{x x}\right)\left(\eta_{x x}+2 u_{x} \eta_{x u}+u_{x}^{2} \eta_{u u}+u_{x x} \eta_{u}-u_{x} \xi_{x x}-\right. \\
\left.-2 u_{x}^{2} \xi_{x u}-2 u_{x x} \xi_{x}-u_{x}^{3} \xi_{u u}-3 u_{x} u_{x x} \xi_{u}\right)+ \\
+2\left(1-x v u_{x x}\right)^{3}\left(r u_{x} \xi+r x\left(\eta_{x}+u_{x} \eta_{u}-u_{x} \xi_{x}-u_{x}^{2} \xi_{u}\right)-r \eta\right)=0 .
\end{gathered}
$$

Equation (1.20) for the case $v \neq 0$ has at $u_{x x}^{3}$ multiplier

$$
\eta_{t}+r x u_{x} \tau^{\prime}(t)-r u\left(\tau^{\prime}(t)-\eta_{u}+u_{x} \xi_{u}\right)-u_{x} \xi_{t}+r u_{x} \xi+r x\left(\eta_{x}-u_{x} \xi_{x}\right)-r \eta,
$$

after its splitting with respect to $u_{x}$, we obtain two equations

$$
\begin{aligned}
& \eta_{t}+r x \eta_{x}+r u \eta_{u}-r \eta-r u \tau^{\prime}(t)=0, \\
& r x \tau^{\prime}(t)-\xi_{t}-r x \xi_{x}-r u \xi_{u}+r \xi=0 .
\end{aligned}
$$

After the splitting with respect to $u_{x}$ of the multiplier at $u_{x x}$ in zero degree it follows that

$$
\begin{gathered}
\xi=A(t, x) u+B(t, x), \\
\eta=A_{x}(t, x) u^{2}+C(t, x) u+D(t, x)
\end{gathered}
$$

and by $(1.21),(1.22)$

$$
\begin{gathered}
2 \eta_{t}-2 r u \tau^{\prime}+2 r u \eta_{u}+2 r x \eta_{x}-2 r \eta+w \eta_{x x}=w \eta_{x x}=0 \\
2 r x \tau^{\prime}-2 r u \xi_{u}-2 \xi_{t}+2 r \xi-2 r x \xi_{x}-2 w \xi_{x x}+4 w \eta_{x u}=-w \xi_{x x}+2 w \eta_{x u}=0 .
\end{gathered}
$$

The last equality implies that

$$
\begin{gathered}
A_{x x}=0, \quad A(t, x)=A_{1}(t) x+A_{0}(t), \quad C(t, x)=\frac{1}{2} B_{x}(t, x)+E(t), \\
\xi=A_{1}(t) x u+A_{0}(t) u+B(t, x), \quad \eta=A_{1}(t) u^{2}+\frac{1}{2} B_{x}(t, x) u+E(t) u+D(t, x) .
\end{gathered}
$$


Then from (1.23) it follows that

$$
\begin{gathered}
B_{x x x}=0, \quad D_{x x}=0, \quad \xi=A_{1}(t) x u+A_{0}(t) u+B_{2}(t) x^{2}+B_{1}(t) x+B_{0}(t), \\
\eta=A_{1}(t) u^{2}+B_{2}(t) x u+\frac{1}{2} B_{1}(t) u+E(t) u+D_{1}(t) x+D_{0}(t) .
\end{gathered}
$$

Now the equality (1.22) implies that $A_{1}(t)=F e^{-r t}, A_{0}(t)$ is a constant,

$$
\begin{gathered}
B_{2}(t)=G e^{-r t}, \quad B_{1}(t)=r \tau(t)+H, \quad B_{0}(t)=J e^{r t}, \\
\xi=F e^{-r t} x u+A_{0} u+G e^{-r t} x^{2}+r \tau(t) x+H x+J e^{r t}, \\
\eta=F e^{-r t} u^{2}+G e^{-r t} x u+\frac{1}{2}(r \tau(t)+H) u+E(t) u+D_{1}(t) x+D_{0}(t) .
\end{gathered}
$$

By (1.21) $D_{1}$ is a constant,

$$
\begin{gathered}
D_{0}(t)=K e^{r t}, \quad E(t)=\frac{1}{2} r \tau(t)+P, \\
\eta=F e^{-r t} u^{2}+G e^{-r t} x u+r \tau(t) u+P u+D_{1} x+K e^{r t} .
\end{gathered}
$$

From (1.16) it follows that $\nu=w_{x}\left(F e^{-r t} x+A_{0}\right) u+S\left(t, x, u_{x}, v, w\right)$.

The coefficient at $u_{x x}$ in equation (1.20) is equated to zero and we obtain the equation

$$
\begin{gathered}
-6 x v \eta_{t}-6 x v\left(r x u_{x} \tau^{\prime}(t)-r u \tau^{\prime}(t)+r u \eta_{u}-r u u_{x} \xi_{u}\right)+w \tau^{\prime}(t)+ \\
+6 x v u_{x} \xi_{t}+w u_{x} \xi_{u}+\nu-2 w \xi_{x}-3 w u_{x} \xi_{u}+2 x v w u_{x} \eta_{x u}+x v w u_{x}^{2} \eta_{u u}- \\
-x v w u_{x} \xi_{x x}-2 x v w u_{x}^{2} \xi_{x u}-6 x v\left(r u_{x} \xi+r x \eta_{x}-r x u_{x} \xi_{x}-r \eta\right)=0 .
\end{gathered}
$$

Let us substitute in it the expressions for $\xi, \eta, \nu$ that were found before, and splitting with respect to the variable $u$ leads to the equations

$$
\begin{gathered}
-2 F e^{-r t} w+w_{x}\left(F e^{-r t} x+A_{0}\right)=0 \\
S=4 G e^{-r t} x w-w \tau^{\prime}+2 r w \tau+2 H w+2 F e^{-r t} x u_{x} w+2 A_{0} u_{x} w .
\end{gathered}
$$

The last of them implies the equalities $\nu_{v}=S_{v}=0$, consequently, by (1.16) we obtain

$$
\nu_{u_{x}}=S_{u_{x}}=2 F e^{-r t} x w+2 A_{0} x w=0, \quad A_{0}=F=0 .
$$

Thus,

$$
\begin{gathered}
\xi=G e^{-r t} x^{2}+r \tau(t) x+H x+J e^{r t}, \\
\eta=G e^{-r t} x u+r \tau(t) u+P u+D_{1} x+K e^{r t}, \\
\nu=4 G e^{-r t} x w-w \tau^{\prime}+2 r w \tau+2 H w .
\end{gathered}
$$

Analogous calculations are made with the coefficient at $u_{x x}^{2}$ in equation (1.20), we obtain the equation

$$
\begin{gathered}
6 x^{2} v^{2}\left(\eta_{t}+r x u_{x} \tau^{\prime}-r u \tau^{\prime}+r u \eta_{u}\right)-x v w\left(\tau^{\prime}-\eta_{u}\right)-6 x^{2} v^{2} u_{x} \xi_{t}+2 v w \xi+2 x w \mu-x v \nu+ \\
+x v w\left(\eta_{u}-2 \xi_{x}\right)+6 x^{2} v^{2}\left(r u_{x} \xi+r x \eta_{x}-r x u_{x} \xi_{x}-r \eta\right)=0
\end{gathered}
$$

that implies the equality $\mu=v\left(H-P-\frac{J}{x} e^{r t}+2 G e^{-r t} x\right)$. Therefore $\mu_{u}=\mu_{w}=0$, and for the case $v^{\prime} \neq 0$ obtain $G=0$ from equation (1.19). Then equation (1.18) implies that $\mu_{x}=0$, hence $J=0$. From equation (1.17) it follows that $\mu_{t}=0$, it corresponds to the resulting formula $\mu=(H-P) v$. Thus, $\tau(t)$ is an arbitrary function,

$$
\begin{gathered}
\xi=H x+r \tau(t) x, \quad \eta=K e^{r t}+D_{1} x+P u+r \tau(t) u, \\
\mu=(H-P) v, \quad \nu=2 H w+\left(2 r \tau(t)-\tau^{\prime}(t)\right) w .
\end{gathered}
$$

Let us formulate the result in the form of theorem. 
Theorem 1. The Lie algebra of infinitesimal generators of the equivalency transformations groups for equation (0.1), is generated by operators

$$
\begin{gathered}
Y_{1}=x \partial_{u}, \quad Y_{2}=e^{r t} \partial_{u}, \quad Y_{3}=x \partial_{x}+u \partial_{u}+2 w \partial_{w}, \quad Y_{4}=x \partial_{x}+v \partial_{v}+2 w \partial_{w} \\
Y_{5}=\tau(t) \partial_{t}+r \tau(t) x \partial_{x}+r \tau(t) u \partial_{u}+\left(2 r \tau(t)-\tau^{\prime}(t)\right) w \partial_{w}
\end{gathered}
$$

when $v^{\prime}, w$ are identically unequal to zero.

Remark 1. It is easy to check that the infinitely-dimensional part of the Lie algebra from Theorem 1 consists of operators of the form $Y_{5}$ only.

The extensions of the operators $Y_{k}, k=1,2,3,4,5$, are

$$
\begin{gathered}
\tilde{Y}_{1}=x \partial_{u}+\partial_{u_{x}}, \quad \tilde{Y}_{2}=e^{r t} \partial_{u}, \quad \tilde{Y}_{3}=x \partial_{x}+u \partial_{u}+2 w \partial_{w}, \\
\widetilde{Y}_{4}=x \partial_{x}+v \partial_{v}+2 w \partial_{w}-u_{x} \partial_{u_{x}}, \quad \widetilde{Y}_{5}=\tau \partial_{t}+r \tau x \partial_{x}+r \tau u \partial_{u}+\left(2 r \tau-\tau^{\prime}\right) w \partial_{w} .
\end{gathered}
$$

Therefore, the kernel of the principal Lie algebras for equation (0.1) is one-dimensional with the basis $Y_{2}$, because the corresponding group only doesn't transform the additional variables $v, w$ and their arguments $t, x, u_{x}$.

Corollary 1. The kernel of the principal Lie algebras for equation (0.1) is spanned by the operator $X_{1}=e^{r t} \partial_{u}$ when $v^{\prime}, w$ are identically unequal to zero.

\section{Group classification}

Consider Lie algebra of projections of operators (1.25) on the subspace of the variables $t, x, u_{x}$, $v, w$, i. e. the algebra generated by

$$
\begin{gathered}
Z_{1}=\partial_{u_{x}}, \quad Z_{2}=v \partial_{v}-u_{x} \partial_{u_{x}} \\
Z_{3}=x \partial_{x}+2 w \partial_{w}, \quad Z_{4}=\tau \partial_{t}+r \tau x \partial_{x}+\left(2 r \tau-\tau^{\prime}\right) w \partial_{w} .
\end{gathered}
$$

It is the direct sum of subalgebras $\left\langle Z_{1}, Z_{2}\right\rangle$ and $\left\langle Z_{3}, Z_{4}\right\rangle$ that corresponds to two different functions $v$ and $w$ and their different arguments. Therefore, the subalgebras can be considered separately.

Nonzero structure constants of $\left\langle Z_{1}, Z_{2}\right\rangle$ are $c_{12}^{1}=-1, c_{21}^{1}=1$. Therefore, the inner automorphisms are $E_{1}: \bar{e}^{1}=e^{1}-e^{2} a_{1}, E_{2}: \bar{e}^{1}=e^{1} e^{a_{2}}$. Here $e^{i}, i=1,2$ are the coefficients at $Z_{i}$ respectively in the basis decomposition of $Z$. If $e^{2} \neq 0$, then $e^{1}=0$ by the acting of $E_{1}$. Therefore the optimal system of one-dimensional subalgebras consists of subalgebras with bases $Z_{1}$ and $Z_{2}$.

In the subalgebra $\left\langle Z_{3}, Z_{4}\right\rangle$ there are no nontrivial inner automorphisms, consequently, the optimal system of one-dimensional subalgebras has a form $\Theta_{1}=\left\{\left\langle Z_{2}\right\rangle,\left\langle b Z_{2}+Z_{4}\right\rangle, b \in \mathbb{R}\right\}$.

For operators $Z$ from optimal systems we calculate the expressions

$$
\left.Z\left(V\left(u_{x}\right)-v\right)\right|_{v=V}=0,\left.\quad Z(W(t, x)-w)\right|_{w=W}=0 .
$$

Note, that if $Z$ contains $Z_{1}$ with a nonzero coefficient and doesn't contain $Z_{2}$, then $v^{\prime}=0$. Such case doesn't correspond to the conditions of Theorem 1 . If an operator $Z$ has nonzero coefficients at $Z_{1}$ and at $Z_{3}$, then by $E_{1}$ the coefficient at $Z_{1}$ can be equated to zero for equivalent operator to $Z$. Therefore, the operator $Z_{1}$ can be excluded from further considerations.

We have

$$
\left.Z_{2}\left(V\left(u_{x}\right)-v\right)\right|_{w=W}=-V-u_{x} V^{\prime}=0, \quad V=\beta / u_{x}
$$


for arbitrary $\beta \in \mathbb{R}$. Further,

$$
\left.Z_{3}(W(t, x)-w)\right|_{w=W}=x W_{x}-2 W=0, \quad W=D(t) x^{2}
$$

for arbitrary function $D(t)$. Finally,

$$
\begin{gathered}
\left(b Z_{3}+\left.Z_{4}(W(t, x)-w)\right|_{w=W}=\tau(t) W_{t}+(r \tau(t)+b) x W_{x}-\left(2 r \tau(t)-\tau^{\prime}(t)+2 b\right) W=0,\right. \\
W=\frac{e^{2 r t+2 b \int \frac{d t}{\tau(t)}}}{\tau(t)} \varphi\left(x e^{-r t-b \int \frac{d t}{\tau(t)}}\right)
\end{gathered}
$$

for arbitrary functions $\varphi \neq 0, \tau \neq 0$.

Optimal system of two-dimensional subalgebras consists of $\left\langle Z_{2}, Z_{3}\right\rangle,\left\langle Z_{2}, b Z_{3}+Z_{4}\right\rangle,\left\langle Z_{3}, Z_{4}\right\rangle$. In the first two cases we have the simultaneous specifications for $v$ and $w$ that are already known. In the last one specification we have the form $W=\gamma x^{2} / \tau(t)$.

For the Lie algebra $\left\langle Z_{2}, Z_{3}, Z_{4}\right\rangle$ the specifications are $V=\beta / u_{x}, W=\gamma x^{2} / \tau(t)$.

For every basis operator from the optimal systems calculate the projection of the corresponding generator of the group of equivalency transformations on the space of the variables $t, x, u$. Then $Z_{2}$ corresponds to $\operatorname{pr}_{(t, x, u)}\left(Y_{4}-Y_{3}\right)=-u \partial_{u}$, for the operator $Z_{3}$ it will be $\operatorname{pr}_{(t, x, u)} Y_{3}=x \partial_{x}+u \partial_{u}$, and $\operatorname{pr}_{(t, x, u)}\left(b Y_{3}+Y_{5}\right)=\tau(t) \partial_{t}+(r \tau(t)+b) x \partial_{x}+(r \tau(t) u+b) \partial_{u}$ corresponds to $b Z_{3}+Z_{4}$. It implies the next theorem.

Theorem 2. Let $v^{\prime}, w$ be identically unequal to zero $i$. Then next assertions are true.

1. The principal Lie algebra of the equation

$$
u_{t}+\frac{w(t, x) u_{x x}}{2\left(1-\beta x \frac{u_{x x}}{u_{x}}\right)^{2}}+r\left(x u_{x}-u\right)=0, \quad \beta \neq 0
$$

is generated by the operators $X_{1}=e^{r t} \partial_{u}, X_{2}=u \partial_{u}$.

2. The principal Lie algebra of the equation

$$
u_{t}+\frac{T^{\prime}(t) e^{2 r t+2 b T(t)} \varphi\left(x e^{-r t-b T(t)}\right) u_{x x}}{2\left(1-x v\left(u_{x}\right) u_{x x}\right)^{2}}+r\left(x u_{x}-u\right)=0, \quad T^{\prime}(t) \neq 0, \quad \varphi(z) \neq 0,
$$

is generated by the operators $X_{1}=e^{r t} \partial_{u}, X_{2}=\frac{1}{T^{\prime}(t)} \partial_{t}+\left(\frac{r}{T^{\prime}(t)}+b\right) x \partial_{x}+\left(\frac{r}{T^{\prime}(t)}+b\right) u \partial_{u}$.

3. The principal Lie algebra of the equation

$$
u_{t}+\frac{T^{\prime}(t) e^{2 r t+2 b T(t)} \varphi\left(x e^{-r t-b T(t)}\right) u_{x x}}{2\left(1-\beta x \frac{u_{x x}}{u_{x}}\right)^{2}}+r\left(x u_{x}-u\right)=0, \quad T^{\prime}(t) \neq 0, \quad \varphi(z) \neq 0, \quad \beta \neq 0,
$$

is generated by the operators

$$
X_{1}=e^{r t} \partial_{u}, \quad X_{2}=u \partial_{u}, \quad X_{3}=\frac{1}{T^{\prime}(t)} \partial_{t}+\left(\frac{r}{T^{\prime}(t)}+b\right) x \partial_{x}+\left(\frac{r}{T^{\prime}(t)}+b\right) u \partial_{u} .
$$

4. The principal Lie algebra of the equation

$$
u_{t}+\frac{D(t) x^{2} u_{x x}}{2\left(1-x v\left(u_{x}\right) u_{x x}\right)^{2}}+r\left(x u_{x}-u\right)=0, \quad D(t) \neq 0,
$$


is generated by the operators

$$
X_{1}=e^{r t} \partial_{u}, \quad X_{2}=x \partial_{x}+u \partial_{u}, \quad X_{3}=\frac{1}{D(t)} \partial_{t}+\frac{r}{D(t)} x \partial_{x}+\frac{r}{D(t)} u \partial_{u} .
$$

5. The principal Lie algebra of the equation

$$
u_{t}+\frac{D(t) x^{2} u_{x x}}{2\left(1-\beta x \frac{u_{x x}}{u_{x}}\right)^{2}}+r\left(x u_{x}-u\right)=0, \quad D(t) \neq 0,
$$

is generated by the operators

$$
X_{1}=e^{r t} \partial_{u}, \quad X_{2}=x \partial_{x}, \quad X_{3}=u \partial_{u}, \quad X_{4}=\frac{1}{D(t)} \partial_{t}+\frac{r}{D(t)} x \partial_{x}+\frac{r}{D(t)} u \partial_{u} .
$$

Remark 2. Theorem 1 and Theorem 2 are valid for the case $r=0$.

\section{Conclusion}

Further Theorem 2 will be applied to the search of exact solutions of the option pricing nonlinear models. Specification $W(t, x)=\sigma^{2} x^{2}$ as partial case of $D(t) x^{2}$ corresponds to the ScönbucherWilmott model, if $r=0$, and to Circar-Papanicolaou model for $r \neq 0$.

\section{REFERENCES}

1. Sircar K.R., Papanicolaou G. General Black-Scholes models accounting for increased market volatility from hedging strategies // Appl. Math. Finance, 1998. Vol. 5. P. 45-82.

2. Black F., Scholes M. The pricing of options and corporate liabilities // J. of Political Economy, 1973. Vol. 81. P. 637-659.

3. Frey R., Stremme A. Market volatility and feedback effects from dynamic hedging // Mathematical Finance, 1997. Vol. 7, no. 4. P. 351-374.

4. Frey R. Perfect option replication for a large trader // Finance and Stochastics. 1998. Vol. 2. P. $115-148$.

5. Jarrow R.A. Derivative securities markets, market manipulation and option pricing theory // J. of Financial and Quantitative Analysis, 1994. Vol. 29. P. 241-261.

6. Schönbucher P., Wilmott P. The feedback-effect of hedging in illiquid markets // SIAM J. on Applied Mathematics, 2000. Vol. 61. P. 232-272.

7. Ovsyannikov L.V. Group analysis of differential equations. New York: Academic press, 1982.

8. Chirkunov Yu.A., Khabirov S.V. Elements of symmetry analysis for differential equations of continuum mechanics. Novosibirsk: Novosibirsk State Technical University, 2012. 659 p. [In Russian]

9. Gazizov R.K., Ibragimov N.H. Lie symmetry analysis of differential equations in finance // Nonlinear Dynamics, 1998. Vol. 17. P. 387-407.

10. Bordag L.A., Chmakova A.Y. Explicit solutions for a nonlinear model of financial derivatives // International Journal of Theoretical and Applied Finance, 2007. Vol. 10, no. 1. P. 1-21.

11. Bordag L.A. On option-valuation in illiquid markets: invariant solutions to a nonlinear model. Mathematical Control Theory and Finance, eds. A. Sarychev, A. Shiryaev, M. Guerra and M. R. Grossinho. Springer, 2008. P. 71-94.

12. Mikaelyan A. Analytical study of the Schönbucher-Wilmott model of the feedback effect in illiquid markets. Master's thesis in financial mathematics, Halmstad: Halmstad University, 2009. viii+67 p.

13. Bordag L.A., Mikaelyan A. Models of self-financing hedging strategies in illiquid markets: symmetry reductions and exact solutions // J. Letters in Mathematical Physics. 2011. Vol. 96, no. 1-3. P. 191-207. 